\title{
Editorial for International Journal of Behavioral Medicine: Special Issue on Cancer
}

\author{
Michael H. Antoni
}

Published online: 12 October 2011

(C) International Society of Behavioral Medicine 2011

\section{Contributions of Behavioral Medicine to Cancer Prevention and Control}

Over the past several decades, behavioral medicine has contributed in many significant ways to cancer prevention and control (CPC) research [1]. This includes primary prevention theory development and behavioral interventions designed to modify cancer risk behaviors tied to exposure to high-fat diet, sexually transmitted viruses, tobacco, and excessive sunlight. This has led to marked decreases in the incidence of lung, skin, colorectal, and cervical cancers. Other work in behavioral medicine has contributed to efforts in secondary prevention by increasing our understanding of processes underlying decisions to undergo cancer screening (mammogram, Pap smears, fecal occult blood testing (FOBT), skin cancer screening) as well as the role of behavioral and psychosocial factors in postscreening follow-up behaviors [1]. This secondary prevention work is designed to reduce mortality as well as psychosocial and physical morbidity [1] and has been widely responsible for the increased use of established screening procedures for breast, cervical, and skin cancers, and is making some gains in increasing screening for prostate, lung, and colorectal cancer. Behavioral medicine research has also extended to examine factors underlying the choice to undergo testing for genetic risk factors such as BRCA 1 and 2, which connote surplus risk for breast and ovarian cancers. This work has led to increased expertise in genetic counseling to guide individuals in the decision-

\section{H. Antoni ( $\bowtie)$}

Department of Psychology and Sylvester Cancer Center,

University of Miami,

5665 Ponce DeLeon Blvd,

Coral Gables, FL 33124, USA

e-mail: Mantoni@miami.edu making challenges (e.g., prophylactic surgery, increased screening frequency) that follow a positive test result.

A huge amount of behavioral medicine research in CPC has been done within the field of "psycho-oncology" mostly targeted to tertiary prevention, wherein behavioral and psychosocial models have been applied to help diagnosed cancer patients optimize psychosocial and physiological adaptation to the stressors of diagnosis, treatment and reentry to their premorbid lives-a period often referred to as early survivorship. The ultimate goal of this work is to enhance quality of life in patients and those connected to them (e.g., family caregivers) as they navigate the cancer experience. This work has applied multiple psychological theories (e.g., cognitive behavioral, existential) to better understand how patients rally their intrapsychic, interpersonal, and spiritual resources to cope with and adapt to the mental and physical challenges of this 1-2-year period. This has resulted in the completion of over 300 randomized trials testing the effects of a wide range of behavioral and psychosocial interventions, which have shown modest but reliable effects on improved quality of life and mood, and reduced side effects (e.g., sleep disruption, fatigue, pain). In a small subset of studies, there is a suggestion that these interventions may help reduce physiologic effects of treatment (compromised immune function) [2]. Very recent work suggests that stress processes may contribute to cancer disease progression, which may help to explain the provocative but difficult to replicate observation that some cancer patients who participate in group-based psychosocial interventions show longer survival times and disease-free intervals [3].

Completing the ways in which behavioral medicine contributes to CPC are quaternary prevention studies that focus on the role of behavioral and psychosocial processes in optimal long-term survivorship after curative and initial 
adjuvant treatments have ended, as well as examining the role of these processes in the end-of-life phase. This behavioral medicine research tests theories about predictors of the development of psychological distress, existential crises, and interpersonal strains on the one hand and improved health surveillance, quality of life, and family functioning on the other as patients maintain their health, or together with their family members move through the terminal phases of the disease. The goals of behavioral medicine interventions in quaternary prevention often span the CPC continuum in terms of reducing cancer risk behaviors/exposures to maintain health, maintaining ongoing screening for recurrent disease, and adapting to physical comorbidities and palliative treatment [1]. It could be said that behavioral medicine research and practice is more comprehensively represented in oncology than in most other medical specialties. The papers included in this special issue of $I J B M$ represent a small subset of some of the most important behavioral medicine research in cancer being done today.

\section{The Special Issue on Behavioral Medicine and Cancer}

The six studies comprising the special issue sample the myriad ways that behavioral medicine contributes across the cancer continuum touching on each level of prevention. These include two studies [4,5] of psychosocial factors underlying cancer screening (secondary prevention) behaviors, three papers focused on tertiary prevention issues in diagnosed patients [6-8], and one paper [9] focused on multiple prevention issues in families dealing with cancer.

The paper by Cole et al. [4] describes a retrospective study designed to identify demographic and psychological predictors of the uptake of colorectal cancer (CRC) screening in South Australia by comparing a set of preventative health model [10]-guided attitudes toward screening in a group of nearly 900 participants and nonparticipants. This work carries significant public health importance given that $\mathrm{CRC}$ is the second most prevalent cancer in developed countries [11], that FOBT screening is associated with a decrease in CRC morality rates [12], and that only $30-58 \%$ of the population may engage in screening $[13,14]$. The study questionnaire was developed through appropriate formative research methods and consisted of 33 items covering 14 health behavior themes. Multivariate analyses revealed that less aversion about and greater value attributed to the FOBT procedure, being in the 65-69-year-old group (vs the 50-54-year-old group), and type of FOBT offered all predicted greater likelihood of undergoing the screen. Although the relative risks of each factor were relatively small, these findings provide an empirically guided set of targets for behavioral interven- tions and alternative specimen sampling procedures designed to increase decisions to pursue CRC screening.

In a second paper focused on cancer screening, de Los Monteros and Gallo [5] provide a detailed review of another attitudinal factor that has been widely studied in the context of decisions to undergo cancer screeningfatalism. The review is unique in its focus on studies relating fatalism to screening behaviors in Latinas, a group for whom cancer fatalism has been documented to be very prevalent and can act as a barrier to successful cancer screening. Latinas are known to have the highest rates of some of the cancers most affected by screening including cervical cancer and also present at later stages for other cancers such as breast and colorectal cancer [15]; all believed to be attributable, in part, to their underutilization of cancer screening [16]. This review therefore addresses an important element of cancer health disparities. A further contribution of the review is an effort to tease apart whether fatalism predicts screening independently of structural barriers (e.g., low access to services and health literacy) in this population. Based upon the 11 articles (on cervical, breast and colorectal screening) that met the strict inclusion criteria, the authors reported that $64 \%$ of the studies demonstrated a significant inverse association between fatalism/cancer fatalism and screening behaviors, even after controlling for structural barriers such as health care access and lower socioeconomic status. The review also illuminates many methodological limitations in this field including limited consensus in operationalizing and measuring fatalism, using self-reported vs objective evidence of screening behaviors, aggregating Latinas across ancestry groups, and a lack of longitudinal designs. This suggests that despite the presence of a reliable association between fatalism and screening behaviors in Latina samples, there remains a need for greater consensus in sampling, measurement, and analytic approaches in order to move the field forward.

The prevalence of depression may approach $45 \%$ in diagnosed cancer patients [17], may have a negative impact on quality of life and treatment nonadherence [18], and is associated with increased mortality rates [19, 20]. Depression is often a major target of cognitive behavior therapy (CBT)-based interventions for cancer patients [21]. Despite this we still know very little about individual difference factors that predict its onset in the period between cancer diagnosis and the completion of curative and initial adjuvant therapies. The paper by Sharpely and colleagues [6] attempts to identify antecedents of depression using the Zung self-rating depression scale (SDS) in 253 Australian women recently diagnosed with breast cancer. The authors hypothesize that different forms of perceived punishmentcomponent 1 , loss of personal or social reinforcement resources and behavioral, and component 2, emotional and 
cognitive reactions to these losses - constitute the phenomenology of depression in recently diagnosed breast cancer patients. They found that $40.1 \%$ of the participants met the clinical criteria for depression, both component 1 and 2 were associated with overall depression scores, and that component 1 was the strongest predictor of overall depression scores and the probability of meeting the clinical criterion for depression. Interestingly the stressor of the breast cancer diagnosis itself was not tapped in the SDS measurement. The authors suggest that the component 2 features of depression (behavioral reactions) may have the function of adaptive coping in response to the component 1 depressive symptoms (perceived losses of sources of reinforcement). These findings suggest that the effectiveness of CBT interventions might be enhanced by identifying the functionality of specific depressive symptoms in the context of recent diagnosis with breast cancer. It is critical to consider these and other nuances of the cancer experience when applying constructs originally derived from populations diagnosed with primary psychiatric conditions. The field would benefit from integrating the use of such functional approaches to measuring depression along with cancer-specific stress and quality of life measures when gauging the appropriateness and efficacy of CBT interventions.

Another tertiary prevention issue that is significant within CPC research concerns factors that relate to successful lifestyle change after a cancer diagnosis. This may involve increasing positive health behaviors such as healthy diet, exercise, and medication adherence as well as eliminating negative behaviors such as tobacco use. Strikingly some studies report that more than half of diagnosed cancer patients continue to smoke after cancer treatment [22]. Although there is a well-established literature identifying attitudinal processes associated with the maintenance and cessation of cigarette smoking in healthy populations, less is known about the predictors of continued smoking and plans for quitting smoking among diagnosed cancer patients, and even less is known about these associations in developing countries. The paper by Schnoll and colleagues [7] makes the case that such knowledge is critical given the established association between tobacco use and negative outcomes in cancer patients [23-25] and the fact that tobacco use is especially prevalent in developing countries such as Russia. They report on a cross-sectional study of attitudinal factors associated with ongoing tobacco use and intentions to quit among 294 current and former smokers newly diagnosed with cancer. The study was guided by the self-medication, social-cognitive, transtheoretical, and neurobiological models of nicotine dependence [26-29]. The results of their analyses suggest that urges to obtain positive reinforcing effects of smoking predict continued smoking, while those intending to quit reported greater perceived risk and self- efficacy for quitting. The authors discuss how these findings can be used to develop behavioral interventions to promote smoking cessation, which in turn could optimize the outcomes of cancer treatment.

As noted above, behavioral and psychosocial models have been applied to helping diagnosed cancer patients optimize quality of life and physical health during the stressful periods of diagnosis, treatment, and survivorship. This has led to the development and demonstrated efficacy of different forms of psychosocial interventions (e.g., stress management, supportive groups) as well as more purely behavioral (e.g., physical exercise, smoking cessation) interventions to improve quality of life and health in these populations [30, 31]. A growing body of research has begun investigating the biobehavioral mechanisms underlying the effects of stress factors as well as psychosocial and behavioral interventions, with a major focus on neuroimmune processes including cellular immune functioning and inflammation [3]. Much of this work has been done in breast cancer patients [2]. In addition to this work, there is an emerging interest in testing the efficacy of physical activity (PA)-based interventions in the context of breast cancer and examining the biobehavioral processes that may underlie their effects on health outcomes. Pakiz and colleagues [8] reason that since obesity is associated with inflammation and inflammation may promote cancer disease progression, PA-based weight loss interventions may improve health outcomes in obese/overweight breast cancer patients by modifying inflammatory cytokines. The authors report on the preliminary effects of a 16-week cognitive behavioral intervention targeting PA and diet in 68 overweight or obese breast cancer survivors. Results suggest that the intervention increased weight loss and PA levels, and was associated with marginally significant decreases in proinflammatory cytokines such as interleukin-6. Although intervention effects on inflammatory biomarkers were modest and the statistical power of the study was limited, there was some evidence that those patients achieving the greatest weight loss showed the greatest decreases in inflammatory measures. This exploratory project may indicate the value of conducting larger scale trials to examine whether such interventions can improve weight management as well as improve quality of life and health outcomes in cancer survivors who are dealing with weight management issues.

The final paper in this special issue addresses the relatively unexplored question of how illness representations about cancer differ among persons who have and have not lived with cancer patients who are going through all the challenges of the disease. Del Castillo and colleagues [9] noted that while illness representations are well-known predictors of disease risk, management, and health outcomes [32, 33], less work has focused on whether persons who have been directly 
exposed to the cancer experience of close family members might change their illness beliefs. With this in mind, they compared illness representations, using the well-established Illness Perception Questionnaire-revised (IPQ-R) [34], among 130 Spanish adults who had vs had not lived with a cancer patient. The results of analyses of the Spanishmodified version of the IPQ-R [35] indicated that those with a family experience with the disease showed greater overall IPQ-R scores, greater identity representations, and a stronger emotional representation than those who had not lived with a cancer patient. The authors suggest that their findings may be used to design tailored prevention interventions that may be helpful in decreasing cancer risk behaviors (primary prevention) and encouraging regular screening (secondary prevention) in healthy persons who have had the unique experience of living with a cancer patient, and may also inform strategies for enhancing family support of diagnosed cancer patients (tertiary and quaternary prevention)

\section{Summary of Special Issue}

The current special issue of $I J B M$ on cancer includes six papers that provide excellent contemporary examples of how behavioral medicine research can be applied to the multiple missions of comprehensive cancer prevention and control. The work presented in each of these papers uses a diverse collection of populations, is solidly grounded in theory, and produces multiple insights that are relevant for public health consideration as well as clinical psychooncology practices. Among the many take-home messages from this collection are: (a) the great need to broaden behavioral medicine research in cancer to countries that have the greatest morbidity and mortality from the disease; (b) the importance and utility of considering the cultural relevance of psychosocial and behavior constructs adapted from health behavior change models; (c) the value of examining underlying biobehavioral and psychosocial mechanisms that can be used to adapt and target interventions; and (d) the mandate to conduct effectiveness trials of those primary, secondary, tertiary, and quaternary prevention interventions that have proven efficacious in controlled settings. All of these considerations will contribute to the successful dissemination of this innovative line of behavioral medicine research in the years to come.

\section{References}

1. Miller S, Bowen D, Croyle R, Rowland J. Overview, current status, and future directions. In: Miller S, Bowen D, Croyle R, Rowland J, editors. Handbook of cancer control and behavioral science. Washington: American Psychological Association; 2009. p. 3-22.
2. McGregor B, Antoni MH. Psychological intervention and health outcomes among women treated for breast cancer: a review of stress pathways and biological mediators. Brain Behav Immun. 2009;23:159-66.

3. Lutgendorf S, Sood A, Antoni MH. Host factors and cancer progression: biobehavioral signaling pathways and interventions. J Clin Oncol. 2010;28:4094-9.

4. Cole S, Zajac I. Gregory T, Mehaffey S., Roosa N, Turnbull D, Esterman A, Young G. Psychosocial variables associated with colorectal cancer screening in South Australia. Int J Behav Med. 2011 (in press).

5. De Los Monteros K, Gallo L. The relevance of fatalism in the study of Latina's cancer screening behavior: A systematic review of the literature. Int J Behav Med. 2011 (in press).

6. Sharpely C, Bitsika V, Christie D. Understanding the functionality of depression among Australian breast cancer patients: Implications for cognitive and behavioural interventions. Int $\mathrm{J}$ Behav Med. 2011 (in press).

7. Schnoll R, Subramanian S, Martinez E, Engstrm P. Correlates of continued tobacco use and intentions to quit smoking among Russian Cancer Patients. Int J Behav Med. 2011 (in press).

8. Pakiz B, Flat S, Bardwell W, Rock C, Mills P. Effects of a weight loss intervention on body mass, fitness and inflammatory biomarkers in overweight or obese breast cancer survivors. Int $\mathrm{J}$ Behav Med. 2011 (in press).

9. Del Castillo A, Godoy-Izquierdo D, Vasquez M, Godoy J. Illness beliefs about cancer among health adults who have and have not lived with cancer patients. Int J Behav Med. 2011 (in press).

10. Myers RE, Ross EA, Jepson C, Wolf TA, Balshem A, Millner L. Modeling adherence to colorectal cancer screening. Prev Med. 1994;23:142-51.

11. Parkin DM, Whelan SL, Ferlay J, Teppo L, Thomas DB. Cancer incidence in five continents. IARC Scientific Publication no.155. Lyon: International Association of Cancer Registries; 2002.

12. Mandel JS, Bond JH, Church TR, Snover DC, Bradley GM, Schuman LM, et al. Reducing mortality from colorectal cancer by screening for fecal occult blood. N Engl J Med. 1993;328:136771.

13. Cole SR, Young GP. Effect of dietary restriction on participation in faecal occult blood test screening for colorectal cancer. Med J Aust. 2001;175:195-8.

14. Australian Institute of Health and Welfare. Screening Monograph No5/2005. The Australian Bowel Cancer Screening Pilot Program: analysis of routinely collected data. Commonwealth of Australia; 2005.

15. American Cancer Society. Cancer Facts and Figures for Hispanics 2006-2008. http://www.cancer.org/downloads/STT/CAFF2006 HispPWSecured.pdf Accessed 20 Jan 2009.

16. Ramirez AG, Suarez L, McAlister A, Villareal R, Trapido E, Talavera GA, et al. Cervical cancer screening in regional Hispanic populations. Am J Health Behav. 2007;24:181-92.

17. Love A, Grabsch B, Clarke D, Bloch S, Kissane D. Screening for depression in women with metastatic breast cancer: a comparison of the Beck depression inventory short form and the hospital anxiety and depression scale. Aust NZ J Psychiatry. 2004;38:526-31.

18. Colleoni M, Mandala M, Peruzzotti G, Robertson C, Bredart A, Goldhirsch A. Depression and degree of acceptance of adjuvant cytotoxic drugs. Lancet. 2000;356:1326-7.

19. Somerset W, Stout S, Miller A, Musselman D. Breast cancer and depression. Oncology. 2004;18:1021-34.

20. Watson M, Haviland J, Greer S, Davidson J, Bliss J. Influence of psychological response on survival in breast cancer: a populationbased cohort study. Lancet. 1999;354:1331-6.

21. Kissane D, Grabsch B, Love A, Clarke D, Bloch S, Smith G. Psychiatric disorder in women with early stage and advanced breast cancer. Aust NZ J Psychiatry. 2004;38:320-6. 
22. Cox LS, Africano NL, Tercyak KP, Taylor KL. Nicotine dependence treatment for patients with cancer. Cancer. 2003;98:632-44.

23. Zhou W, Heist RS, Liu G, Park S, Neuberg DS, Asomaning K, et al. Smoking cessation before diagnosis and survival in early stage nonsmall cell lung cancer patients. Lung Cancer. 2006;53:375-80.

24. Duarte RL, Luiz RR, Paschoal ME. The cigarette burden (measured by the number of pack-years smoked) negatively impacts the response rate to platinum-based chemotherapy in lung cancer patients. Lung Cancer. 2008;61:244-54.

25. Daniel M, Keefe FJ, Lyna P, Peterson B, Garst J, Kelley M. Persistent smoking after a diagnosis of lung cancer is associated with higher reported pain levels. J Pain. 2009;10:323-8.

26. Markou A, Kosten TR, Koob GF. Neurobiological similarities in depression and drug dependence: a self-medication hypothesis. Neuropsychopharmacology. 1998;18:135-74.

27. Lev EL. Bandura's theory of self-efficacy: applications to oncology. Sch Inq Nurs Pract. 1997;11:21-37.

28. Prochaska JO, DiClemente CC, Norcross JC. In search of how people change: applications to addictive behaviors. Am Psychol. 1992;47:1102-14.

29. Koob GF, Le Moal M. Drug addiction, dysregulation of reward, and allostasis. Neuropsychopharmacology. 2001;24:97-129.
30. Antoni MH, Wimberly S, Lechner S, et al. Stress management intervention reduces cancer-specific thought intrusions and anxiety symptoms among women undergoing treatment for breast cancer. Am J Psychiatry. 2006;163:1791-7.

31. Andersen B, Yang H, Farrar W, et al. Psychologic intervention improves survival for breast cancer patients: a randomized clinical trial. Cancer. 2008;113:3450-8.

32. Leventhal H, Leventhal EA, Cameron L. Representations, procedures, and affect in illness self-regulation: a perceptualcognitive model. In: Baum A, Revenson TA, Singer JE, editors. Handbook of health psychology. Mahwah: Lawrence Erlbaum; 2001. p. 19-48.

33. Leventhal H, Brissette I, Leventhal EA. The common-sense model of regulation of health and illness. In: The self-regulation of health and illness behaviour. London: Routledge; 2003. p. 42-65.

34. Moss-Morris R, Weinman J, Petrie K, Horne R, Cameron L, Buick D. The revised Illness Perception Questionnaire (IPQ-R). Psychol Health. 2002;17:1-16.

35. Beléndez R, Bermejo RM, García-Ayala MD. Estructura factorial de la versión española del Revised Illness Perception Questionnaire en una muestra de hipertensos. Psicothema. 2005;17:318 24. 